\title{
BENEFÍCIOS DA PRÁTICA DE ATIVIDADE FÍSICA AO AR LIVRE
}

DOI: $10.48140 /$ digitaleditora.2020.002.20

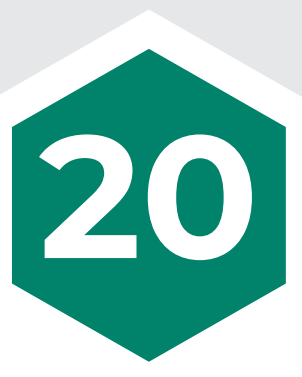

RESUMO

Objetivos: Esse trabalho teve por objetivo fazer a investigação dos benefícios da prática de atividades física ao ar livre para seus praticantes.

Métodos: Para isso, foi desenvolvida uma revisão de literatura através das bases de dados acadêmicos Scientific Electonic Library OnLine (SCIELO), Google Acadêmico, National Library of Medicene (PubMed) e Centro Latino-Americano e do Caribe de Informações em Ciências da Saúde (BIREME).

Resultados: Para encontrar artigos voltados para temática foram usados os seguintes descritores: atividade física, ar livre, benefícios, parques.

Conclusão: O trabalho concluiu que a prática de atividade física ao ar livre possibilita, além de benefícios acerca da saúde física, também abarca a mental e a socialização de seu praticante. 


\section{BENEFITS OF OUTDOOR PHYSICAL ACTIVITY PRACTICE}

DOI: 10.48140/digitaleditora.2020.002.20

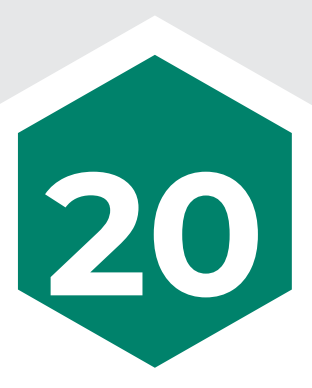

ABSTRACT

Recebido em: 10/12/2020

Aprovado em: 28/12/2020

Conflito de Interesse: não

Suporte Financeiro: não houve

Objectives: This work aimed to investigate the benefits of practicing physical activities outdoors for its practitioners.

Methods: For this, a literature review was developed through the academic databases Scientific Electonic Library OnLine (SCIELO), Google Scholar, National Library of Medicene (PubMed) and Latin American and Caribbean Center on Health Sciences Information ( BIREME).

Results: To find articles focused on the theme, the following descriptors were used: physical activity, outdoors, benefits, parks. Conclusion: The work concluded that the practice of physical activity outdoors allows, in addition to benefits about physical health, also encompasses the mental and socialization of its practitioner. 


\section{INTRODUÇÃO}

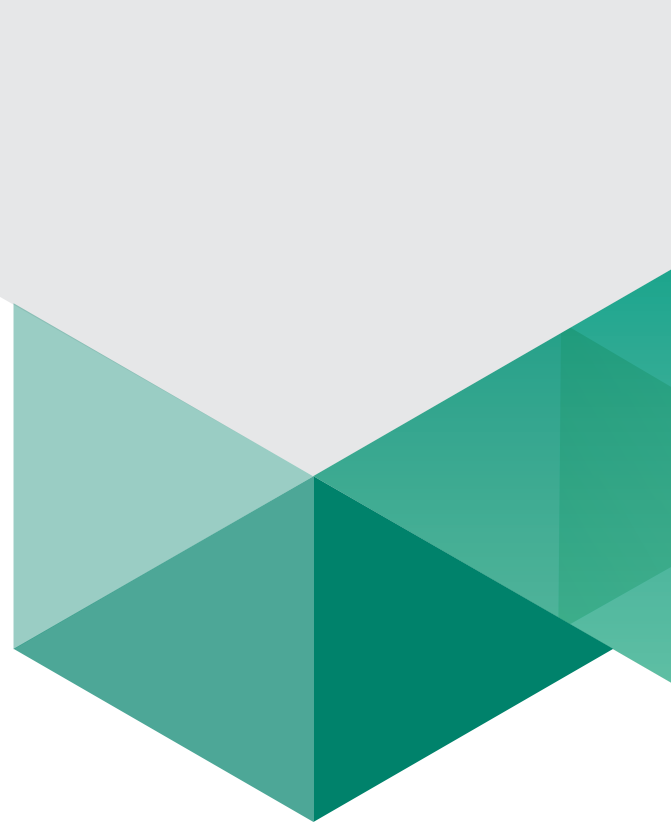

Para a Organização Mundial da Saúde (OMS), atividade física pode ser definida como sendo qualquer movimento corporal produzido pelos músculos esqueléticos que requeiram gasto de energia incluindo atividades praticadas durante o trabalho, jogos, execução de tarefas domésticas, viagens e em atividades de lazer (OMS, 2010). É reconhecido pela literatura, que essa condição metabólica, é um componente importante para proteção e promoção da saúde, promovendo diversos benefícios, como: melhoria da capacidade cardiovascular, prevenção da obesidade, melhoria na capacidade cardiorrespiratória, controle da taxa de glicose e lipídicas, melhoria da mineralização óssea (GOLDNER, 2013; PEDRINELLI et al., 2009; RODRIGUES, 2013). Entretanto, são observados aspectos ampliados quando esses estímulos são sistematizados e respeitam condições de preferências individuais e intensidades específicas para cada público (NETTO, 2012).

A atividade física também se apresenta como um importante aliado para fatores cognitivos e emocionais, no combate dos fatores associados a depressão, aumento dos níveis de humor, disposição, capacidade de aprendizagem e autoestima. Além de reduzir os níveis de ansiedade e estresse (GOLDNER, 2013).

Nesta perspectiva, praticar atividades físicas regulares são imprescindíveis para que sejam restabelecidas a saúde em face dos danos que o cotidiano estressante no ciclo de vida dos indivíduos, sobretudo em ambientes mais democráticos (MORAES et al., 2009).

Entretanto, na contramão das inúmeras evidências, é reconhecido na literatura, que uma parcela importante da população se encontra com níveis abaixo dos recomendados para manutenção dos níveis de saúde, especialmente o público jovem, e em destaque, o sexo feminino. (OMS, 2008). É importante ressaltar, que essa projeção de crescimento estatístico se encontra em ascensão, sobretudo nas últimas décadas (OMS, 2016).

Nessa perspectiva, tem motivado o aumento crescente da atenção à saúde, direcionando as políticas públicas, principalmente, na construção de praças públicas e parques nas cidades. Assim, tem sido valorizado o uso desses espaços pela população em geral para a prática de atividades físicas. Adicionalmente, é importante ressaltar, que estes espaços possuem fácil acesso, baixo custo e são reconhecidos, como, ambientes de manutenção da saúde física, de socialização e de lazer. Nessa perspectiva, se beneficiar desses espaços para a prática de atividades físicas, tem o potencial de promover 
melhoria na qualidade de vida da população, possibilitando a prevenção da saúde, além de ampliar as possibilidades de socialização dos seus frequentadores entre outros aspectos associados (SILVA et al., 2015).

Pesquisas apontam que atividades físicas praticadas ao ar livre se apresentam como excelentes possibilidades para o indivíduo que busca uma melhor qualidade de vida. Para além de trazer benefícios físicos, esse tipo de modalidade também é recomendada para o investimento em uma saúde mental e reconhecida por otimizar a exposição solar e síntese de vitamina D. Como também, contribuir com a frequência e adesão pela flexibilidade e autonomia que geralmente estão atreladas a essas práticas corporais (ZAITUNE et al., 2010).

Posto isto, é necessário que estudos acerca da temática sejam produzidos e vinculados com o objetivo de informar à sociedade as diversas possibilidades de práticas de atividade física ao ar livre e sobretudo, sem a necessidade de altos investimentos. O objetivo do estudo é apresentar os benefícios proporcionados pela prática da atividade física ao ar livre.

O estudo tem caráter qualitativo, tal pesquisa, é de importante relevância em estudos que tenha foco no social, visto que há perceptível aumento nas condicionantes acerca do que está sendo observado. O trabalho apresenta a metodologia da pesquisa bibliográfica, para isto, foi realizado levantamento de dados através de plataformas acadêmicas. Os campos de busca foram: Scientific Electonic Library OnLine (SCIELO), Google Acadêmico, National Library of Medicene (PubMed) e Centro Latino-Americano e do Caribe de Informações em Ciências da Saúde (BIREME). Foram eleitos para compor o estudo, exclusivamente artigos que apontassem para o objetivo da pesquisa. Ademais, foram selecionados apenas artigos no modelo de ensaios clínicos, no idioma português, disponíveis nas bases de dados e respeitando o período cronológico de 2002 à 2020, procurando, deste modo, manter fontes atualizadas sobre o tema em questão. Para encontrar artigos voltados para temática foram feitas associações com os seguintes descritores:

$1^{\circ}$ associação: atividade física e benefícios metabólicos.

$2^{\circ}$ associação: atividade física e ar livre.

$3^{\circ}$ associação: atividade física e saúde mental.

A análise foi feita através da proximidade do objetivo da pesquisa e títulos. Posteriormente, foram realizadas, as leituras dos artigos encontrados, separando-os em primeira fase pela leitura do resumo e, posteriormente, leitura na íntegra dos artigos selecionados frente a coerência com os objetivos do estudo. 


\section{RESULTADOS E DISCUSSÃO}

Seguindo o método descrito e considerando as palavras chaves e sua disposição frente ao processo de consulta da base de dados e triagem, foram incluídos 24 artigos, como representados na (Figura 1). Não foram incluídos artigos da base de dados (PubMed), depois do crivo do processo metodológico de mapeamento e eleição dos artigos para a pesquisa.

FIGURA 1.

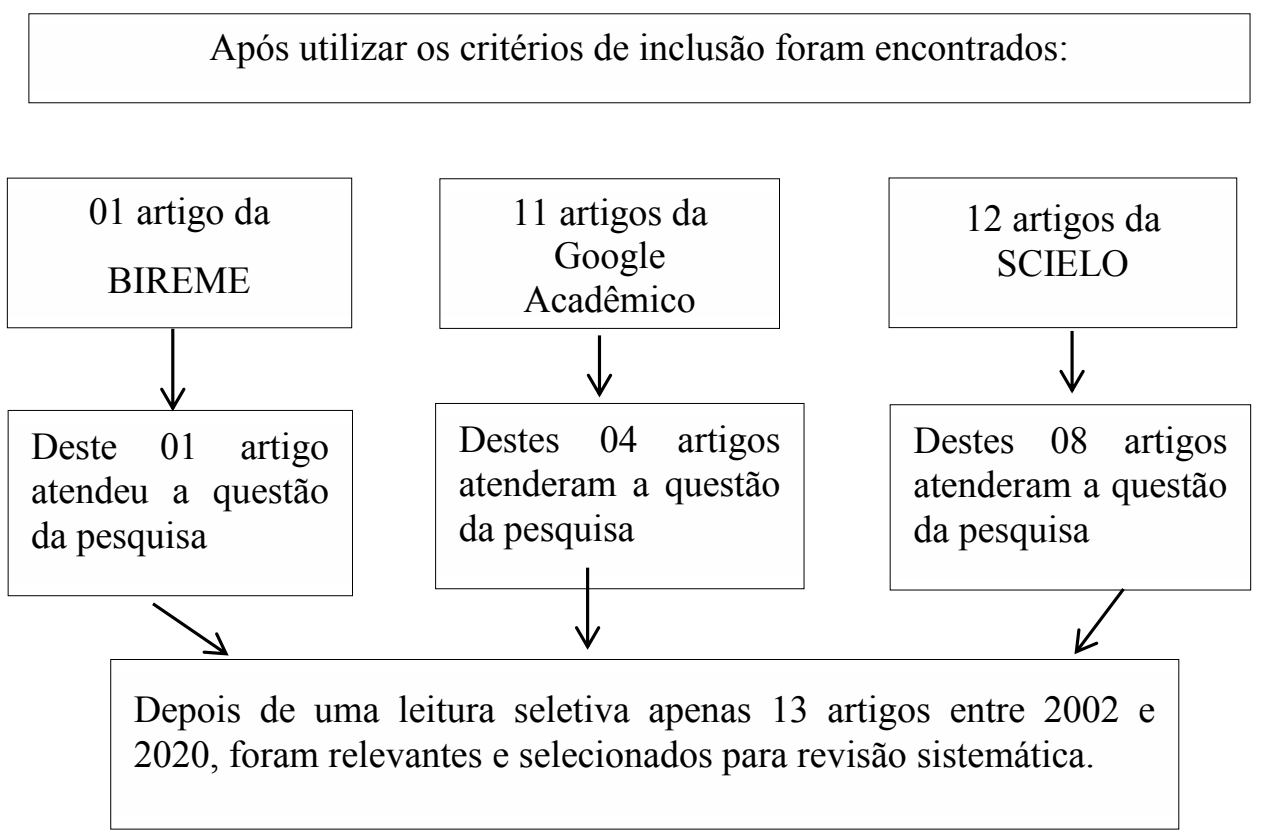

Conforme a pesquisa, foi amplamente exposto, por meio da literatura indexada nas bases de dadas da pesquisa, que a atividades física ao ar livre está intimamente atrelada a inúmeros benefícios aos seus praticantes. Ademais, esses benefícios independem da classe social, sexo ou idade (MACIEL, 2010).

No que diz respeito aos aspectos relacionados ao controle emocional e metabólicos, a prática de atividades físicas possibilita benefícios acerca da autoimagem e autoestima de quem a prática, que fisicamente possui relação com a diminuição de doenças cardiovasculares, de mortes prematuras, diminuição dos casos de acidente vascular cerebral, diabetes mellitus tipo II.

Segundo Gomes Júnior et al. (2015), praticar atividade física regularmente proporciona diversos benefícios, independente da faixa etárias, trazendo melhoria da capacidade funcional e da habilidade física desses sujeitos, possibilitando a socialização, a longevidade, causando a melhora dos níveis de humor e do estado mental.

Ademais, a atividade física, é compreendido como uma forma de lazer que tem o objetivo de reduzir o estresse presente no cotidiano dos sujeitos, onde uma se apresenta como uma agradável e viciante atividade que diminui sensações de desconforto como irritabilidade, ansiedade e depressão, 
além da manutenção de forma física boa e melhoria da qualidade de vida (SILVA et al., 2010).

A atualidade, a inatividade física é um risco significativo para a saúde. Um indivíduo que é compreendido como inativo fisicamente é o que tem em sua rotina diária a quantidade mínima de atividade física, com um gasto energético melhor semanal menor que $500 \mathrm{kcal}$ provenientes de atividades rotineiras do cotidiano (MACIEL, 2010).

Sendo esses responsáveis por refletir de forma direta no nível de saúde desses sujeitos. Na atualidade, é crescente, a conscientização da população ao que diz respeito do quão é importante usar os espaços ao ar livre para a prática de atividades de lazer e físicas, possibilitando a preservação ambiental e a convivência social nos grandes centros urbanos (DE SOUZA et al., 2017).

Com efeito da acessibilidade e a beleza natural dos parques públicos, estes locais tornaram-se ambientes de saúde e bem-estar, que são fundantes para a prevenção à agravos à saúde e de doenças, além de proporcionar diversão e lazer para as pessoas que fazem uso deles (PIERONE, 2016).

Implantar esse tipo de local próximos a regiões residenciais proporcionar aos moradores das comunidades próximas uma área que possibilita a saúde, que favorece um estilo de vida que seja mais saudável (ativo) para esta população (DE SOUZA et al., 2017).

Os parques públicos têm se tornado uma referência no tocante da saúde, pelo fato de possibilitarem a interação entre o meio ambiente e homem, que em conjunto com a prática de atividades físicas resultam em benefícios físicos, sociais e psicológicos (PIERONE, 2016).

Áreas verdes, praças e ciclovias são exemplos de áreas que têm um importante papel na prática de atividades físicas ao ar livre, possibilitando um estilo de vida mais saudável (ativo) para a população dos centros urbanos (SILVA et al., 2015).

Salienta-se que por meio da prática de atividades físicas ao ar livre, sejam direcionadas políticas públicas de combate ao sedentarismo e a todos os malefícios combinados, especialmente, frente as mudanças no estilo de vida e comportamentais das últimas décadas. Embora seja reconhecido que a adesão e permanência a prática de atividade física, tenha aspectos multifatoriais (MACIEL, 2010). As atividades físicas ao ar livre, mostram-se como uma alternativa mais flexível, de menores custos e investimentos para início da prática. Contribuindo com a afirmativa, a caminhada se apresenta como um acessível estilo de atividade física, que pode ter sua realização em áreas verdes e espaços públicos, em face de que esta atividade não precisa da utilização de equipamentos complexos e por meio dela é possível causar o estimulo à população para que se torne mais ativa fisicamente (KUNZLER et al., 2014). 


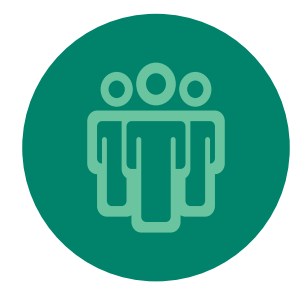

\section{CONCLUSÃO}

Após realização do estudo os resultados são consistentes e fornecem dados importantes frente aos benefícios metabólicos e sociais da atividade física ao ar livre e sua praticidade, possibilidades e abrangência.

A atividade física pratica ao ar livre, além de evitar o sedentarismo traz contribuições para a socialização do indivíduo, aumento da autoestima, melhoria do humor, além de causar a diminuição da sensação de obrigação em estar na academia. Posto isso, o presente trabalho conclui que a prática de atividades físicas ao ar livre evita o sedentarismo e outros problemas de saúde, entendidos como agravos sérios tanto física como mentalmente. Nessa condição, mostra-se, como uma importante ferramenta para direcionar as políticas públicas de atenção à saúde. 


\section{REFERÊNCIAS}

DE SOUZA, R.G. et al. A influência da prática da atividade Física ao ar livre no desenvolvimento social de Capitais do nordeste. Caderno de Graduação-Ciências Biológicas e da Saúde-UNIT-SERGIPE, v. 4, n. 1, p. 77, 2017.

GOLDNER, L.J. Educação física e saúde: benefício da atividade física para a qualidade de vida. 2013. 24 f. TCC (Graduação)- Curso de Educação Física, Centro Educação Física e Desporto- Cefd, Universidade Federal do Espirito Santo - Ufes. 2013.

GOMES JÚNIOR, V.F.F. et al. Compreensão de idosos sobre os benefícios da atividade física. Rev. bras. ciênc. saúde, p. 193-198, 2015.

KUNZLER, M.R. et al. Saúde no parque: características de praticantes de caminhada em espaços públicos de lazer. Saúde em debate, v. 38, p. 646-653, 2014.

MACIEL, M.G. Atividade física e funcionalidade do idoso. Motriz: Revista de Educação Física. 2010; v. 16, n.4, p. 1024-32.

MORAES, A.C.F. de et al. Prevalência de inatividade física e fatores associados em adolescentes. Revista da Associação Médica Brasileira, v. 55, n. 5, p. 523-528, 2009.

NETTO, R.S.M. Nível de atividade física e qualidade de vida de estudantes universitários da área da saúde. Rev Bras de Ciências da Saúde. 2012;10(34):47-55.

OMS. Organização Mundial da Saúde. 2008; 2010; 2016.

PEDRINELLI, A. et al. O efeito da atividade física no aparelho locomotor do idoso. Revista Brasileira de Ortopedia, v. 44, n. 2, 2009

PIERONE, J.M. et al. Qualidade de vida de usuários de parques públicos. Boletim de psicologia, v. 66, n. 144, p. 99-112, 2016.

RODRIGUES, F.J.L. Comparação da densidade mineral óssea entre indivíduos com esquizofrenia e indivíduos saudáveis: relação com a medicação antipsicótica e o nível de atividade física. Dissertação ( Mestrado em Ciências do Desporto) Faculdade de Desporto Universidade do Porto. 2013.

SILVA, R.S. et al. Atividade física e qualidade de vida. Ciência \& Saúde Coletiva v.15, n.1, pp.115-120, 2010.

SILVA, I. et al. Espaços públicos de lazer: distribuição, qualidade e adequação à prática de atividade física. Revista Brasileira de Atividade Física \& Saúde, v. 20, n. 1, p. 82-82, 2015.

ZAITUNE, M.P.A. et al. Fatores associados à prática de atividade física global e de lazer em idosos: Inquérito de Saúde no Estado de São Paulo (ISA-SP), Brasil. Cadernos de Saúde Pública, v. 26, p. 16061618, 2010. 